\title{
Research of Firing Control Model of Multi-stage Synchronous Induction Coil Launcher
}

\author{
Keyi Zhao ${ }^{1,2}$, Xichao Yuan ${ }^{1,}$, Hongjun Xiang ${ }^{1}$, Qian Zhang ${ }^{1}$, Qingao Lv ${ }^{1}$ \\ ${ }^{1}$ Shijiazhuang Campus, Army Engineering University, Shijiazhuang, Hebei 050003 China \\ ${ }^{2}$ Hebei Jiaotong Vocational And Technical College Department of Electric and Information Engineering, Shijiazhuang, Hebei 050003 \\ China \\ * Corresponding author
}

\begin{abstract}
The problem of firing control is a critical technology of multi-stage synchronous induction coil launcher (MSSICL). Operation with high launch efficiency of MSSICL mainly depends on each stage driving coil energized when the armature moves on the proper location. In order to solve the problem of firing control for MSSICL, the firing control model of MSSICL is built based on the dynamically simulative model and the firing control criterion. The dynamically simulative model includes the voltage equations of driving circuit and the kinematic equations. The solved method is provided. In addition, the factors that influence the solved precision of the firing control model of MSSICL are analyzed.
\end{abstract}

\section{INTRODUCTION}

Multi-stage driving coils are fed at given time sequence and the armature is accelerated in the axial direction of multi-stage synchronous induction coil launcher (MSSICL). In order to effectively accelerate the armature, energized time of each stage need approximately keeping synchronous with the armature's location [1 3]. The determination of energized time of each stage or energized location of the armature relative with each stage is very critical. The method of the armature location signal measured timely as the start signal of the driving coil energized is feasible in low velocity EM mortar $[4,5]$. But it may be not feasible for high velocity MSSICL because the systems of the armature location measurement and the signal process have some response time. If the response time is relatively long, high effective operation is impossible to achieve.

In order to solve the firing control problem of MSSICL, firing control model (FCM) is built based on dynamically simulative model and (DSM) firing control criterion (FCC). The solved method is provided. These provide the theoretical foundation for the development of the firing control soft for MSSICL.

\section{EQUIVALENT CIRCUIT MODEL OF MSSICL}

Considering the eddy distribution in the solid armature, it is subdivided into concentric hoops with rectangular cross-section, and the eddy in each of them is assumed to be uniform. But the driving coil can be regarded as a single element because the driving current distribution is uniform. Each filament armature loop is composed of its resistance Raj and its self-inductance Laj. Each driving circuit excepting for the driving coil includes stored energy capacitor $\mathrm{Ci}$, main switch $\mathrm{Si}$, crowbar diode $\mathrm{Di}$ and crowbar resistance Rbi. The structure and the equivalent circuit model of MSSICL are shown in Figure 1.
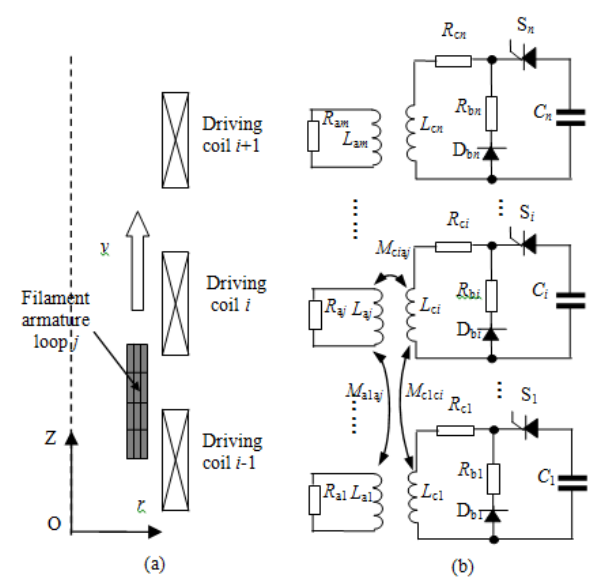

Figure 1. Structure model and equivalent circuit model of MSSICL. Fig.1 (a) indicates the structure mode, and Fig.1 (b) indicates the equivalent circuit model.

$\mathrm{D}_{i}$ and $R_{\mathrm{b} i}$ are composed of the crowbar branch. According to the working status of the crowbar branch, the driving circuit can be divided into two phases. The first phase is that the crowbar branch does not work, and the second phase is that the crowbar branch works. In practical working process, it is possible for some driving

a Corresponding author: angell_chaser@qq.com 
circuits in the first phase and for some others in the second phase.

Driving circuit at the first phase belongs to $R L C$ circuit. Exchanged condition of the crowbar branch is that $C_{i}$ completes the discharge in and is charged in reverse direction. Driving circuit at the second phase belongs to $R L$ circuit.

\section{BUILDING OF FIRING CONTROL MODEL}

It is necessary to build FCM through the combination of DSM and FCC.

\subsection{Dynamically simulative model of MSSICL}

Assumed that the stage number of the driving coils is $n$ and the number of the filament armature loop is $m$, the loop voltage equations before exchanged can be expressed as

$$
\left\{\begin{array}{c}
U_{\mathrm{c} 1}=R_{\mathrm{c} 1} i_{\mathrm{c} 1}+\sum_{i=1}^{n}\left(M_{\mathrm{clci} i} \frac{\mathrm{d} i_{\mathrm{c} i}}{\mathrm{~d} t}\right)+\sum_{j=1}^{m}\left(\frac{\mathrm{d}\left(M_{\mathrm{claj}} i_{\mathrm{aj}}\right)}{\mathrm{d} t}\right) \\
U_{\mathrm{c} 2}=R_{\mathrm{c} 2} i_{\mathrm{c} 2}+\sum_{i=1}^{n}\left(M_{\mathrm{c} 2 \mathrm{c} i} \frac{\mathrm{d} i_{\mathrm{c} i}}{\mathrm{~d} t}\right)+\sum_{j=1}^{m}\left(\frac{\mathrm{d}\left(M_{\mathrm{c} 2 \mathrm{aj}} i_{\mathrm{aj} j}\right)}{\mathrm{d} t}\right) \\
\vdots \\
U_{\mathrm{c} n}=R_{\mathrm{cn}} i_{\mathrm{cn}}+\sum_{i=1}^{n}\left(M_{\mathrm{cnci}} \frac{\mathrm{d} i_{\mathrm{c} i}}{\mathrm{~d} t}\right)+\sum_{j=1}^{m}\left(\frac{\mathrm{d}\left(M_{\mathrm{cna} j} i_{\mathrm{aj} j}\right)}{\mathrm{d} t}\right) \\
0=R_{\mathrm{a} 1} i_{\mathrm{a} 1}+\sum_{j=1}^{m}\left(M_{\mathrm{a} 1 \mathrm{aj} j} \frac{\mathrm{d} i_{\mathrm{a} j}}{\mathrm{~d} t}\right)+\sum_{i=1}^{n}\left(\frac{\mathrm{d}\left(M_{\mathrm{cia} 1} i_{\mathrm{ci} i}\right)}{\mathrm{d} t}\right) \\
\vdots \\
0=R_{\mathrm{a} m} i_{\mathrm{a} m}+\sum_{j=1}^{m}\left(M_{\mathrm{amaj}} \frac{\mathrm{d} i_{\mathrm{a} j}}{\mathrm{~d} t}\right)+\sum_{i=1}^{n}\left(\frac{\mathrm{d}\left(M_{\mathrm{ciam}} i_{\mathrm{c} i}\right)}{\mathrm{d} t}\right)
\end{array}\right.
$$

Equation (1) can be described by the matrix form as

$$
U=R I_{1}+M_{1} \dot{I}_{1}+M_{2} \dot{I}_{2}+v \hat{M} I_{2}
$$

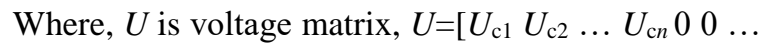

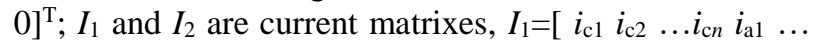
$\left.i_{\mathrm{a} m}\right]^{\mathrm{T}}, I_{2}=\left[\begin{array}{lllllll}i_{\mathrm{a} 1} & \ldots & i_{\mathrm{a} m} & i_{\mathrm{c} 1} & i_{\mathrm{c} 2} & \ldots & i_{\mathrm{c} n}\end{array}\right]^{\mathrm{T}} ; \dot{I}_{1}$ and $\dot{I}_{2}$ are current differential coefficient matrixes,

$$
\begin{gathered}
\dot{I}=\left[\begin{array}{lllllll}
\frac{\mathrm{d} i_{\mathrm{c} 1}}{\mathrm{~d} t} & \frac{\mathrm{d} i_{\mathrm{c} 2}}{\mathrm{~d} t} & \cdots & \frac{\mathrm{d} i_{\mathrm{c} n}}{\mathrm{~d} t} & \frac{\mathrm{d} i_{\mathrm{a} 1}}{\mathrm{~d} t} & \cdots & \frac{\mathrm{d} i_{\mathrm{a} m}}{\mathrm{~d} t}
\end{array}\right]^{\mathrm{T}}, \\
\dot{I}_{2}=\left[\begin{array}{lllllll}
\frac{\mathrm{d} i_{\mathrm{a} 1}}{\mathrm{~d} t} & \frac{\mathrm{d} i_{\mathrm{a} 2}}{\mathrm{~d} t} & \cdots & \frac{\mathrm{d} i_{\mathrm{a} m}}{\mathrm{~d} t} & \frac{\mathrm{d} i_{\mathrm{c} 1}}{\mathrm{~d} t} & \cdots & \frac{\mathrm{d} i_{\mathrm{c} n}}{\mathrm{~d} t}
\end{array}\right]^{\mathrm{T}} ;
\end{gathered}
$$

$v$ is the armature's speed, $R$ is resistance matrix, $\hat{M}$ is mutual gradient matrix,

$$
R=\left[\begin{array}{ccccccc}
R_{\mathrm{c} 1} & 0 & \cdots & 0 & 0 & \cdots & 0 \\
0 & R_{\mathrm{c} 2} & \cdots & 0 & 0 & \cdots & 0 \\
\vdots & \vdots & \ddots & \vdots & \vdots & \cdots & \vdots \\
0 & 0 & \cdots & R_{\mathrm{cn}} & 0 & \cdots & 0 \\
0 & 0 & \cdots & 0 & R_{\mathrm{a} 1} & \cdots & 0 \\
\vdots & \vdots & \ddots & \vdots & \vdots & \ddots & \vdots \\
0 & 0 & \cdots & 0 & 0 & \cdots & R_{\mathrm{a} m}
\end{array}\right] ;
$$

$M_{1}$ and $M_{2}$ are mutual matrixes,

$$
\begin{aligned}
& M_{1}=\left[\begin{array}{cccccccc}
M_{\mathrm{clc1}} & M_{\mathrm{clc2}} & \cdots & M_{\mathrm{clcn}} & 0 & 0 & \cdots & 0 \\
M_{\mathrm{c} 2 \mathrm{c} 1} & M_{\mathrm{c} 2 \mathrm{c} 2} & \cdots & M_{\mathrm{c} 2 c n} & 0 & 0 & \cdots & 0 \\
\vdots & \vdots & \vdots & \vdots & \vdots & \vdots & \vdots & \vdots \\
M_{\mathrm{cnc1}} & M_{\mathrm{cnc2}} & \cdots & M_{\mathrm{c} n c n} & 0 & 0 & \cdots & 0 \\
0 & 0 & \cdots & 0 & M_{\mathrm{a} 1 \mathrm{a} 1} & M_{\mathrm{a} 1 \mathrm{a} 2} & \cdots & M_{\mathrm{alam}} \\
\vdots & \vdots & \vdots & \vdots & \vdots & \vdots & \vdots & \vdots \\
0 & 0 & \cdots & 0 & M_{\text {amal }} & M_{\text {ama2 }} & \cdots & M_{\text {amam }}
\end{array}\right], \\
& M_{2}=\left[\begin{array}{cccccccc}
M_{\text {cla1 }} & M_{\text {cla2 }} & \cdots & M_{\text {clam }} & 0 & 0 & \cdots & 0 \\
M_{\text {c2a1 }} & M_{\text {c2a2 }} & \cdots & M_{\text {c2am }} & 0 & 0 & \cdots & 0 \\
\vdots & \vdots & \vdots & \vdots & \vdots & \vdots & \vdots & \vdots \\
M_{\text {cnal }} & M_{\text {cna2 }} & \cdots & M_{\text {cnam }} & 0 & 0 & \cdots & 0 \\
0 & 0 & \cdots & 0 & M_{\text {clal }} & M_{\text {c2al }} & \cdots & M_{\text {cnal }} \\
\vdots & \vdots & \vdots & \vdots & \vdots & \vdots & \vdots & \vdots \\
0 & 0 & \cdots & 0 & M_{\text {clam }} & M_{\text {c2am }} & \cdots & M_{\text {cram }}
\end{array}\right] ;
\end{aligned}
$$

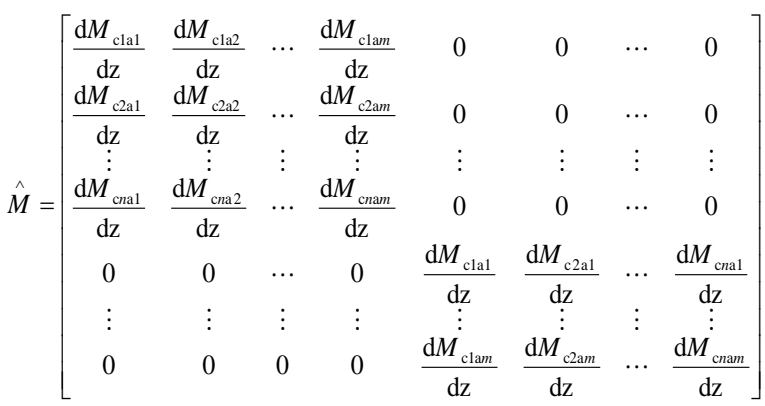

Under the condition of ignoring the voltage drop of crowbar diode, the loop voltage equations after exchanged for all stages can be expressed as

$$
\begin{aligned}
& 0=R_{\mathrm{c} 1} i_{\mathrm{c} 1}+\sum_{i=1}^{n}\left(M_{\mathrm{clci}} \frac{\mathrm{d} i_{\mathrm{c} i}}{\mathrm{~d} t}\right)+\sum_{j=1}^{m}\left(\frac{\mathrm{d}\left(M_{\mathrm{claj}} i_{\mathrm{aj}}\right)}{\mathrm{d} t}\right)+R_{\mathrm{b} 1} i_{\mathrm{c} 1} \\
& 0=R_{\mathrm{c} 2} i_{\mathrm{c} 2}+\sum_{i=1}^{n}\left(M_{\mathrm{c} 2 c i} \frac{\mathrm{d} i_{\mathrm{c} i}}{\mathrm{~d} t}\right)+\sum_{j=1}^{m}\left(\frac{\mathrm{d}\left(M_{\mathrm{c} 2 \mathrm{aj}} i_{\mathrm{aj}}\right)}{\mathrm{d} t}\right)+R_{\mathrm{b} 2} i_{c 2} \\
& \left\{0=R_{\mathrm{c} n} i_{\mathrm{cn}}+\sum_{i=1}^{n}\left(M_{\mathrm{cnci}} \frac{\mathrm{d} i_{\mathrm{c} i}}{\mathrm{~d} t}\right)+\sum_{j=1}^{m}\left(\frac{\mathrm{d}\left(M_{\mathrm{cnaj}} i_{\mathrm{aj}}\right)}{\mathrm{d} t}\right)+R_{\mathrm{b} n} i_{\mathrm{cn}}\right. \\
& 0=R_{\mathrm{al}} i_{\mathrm{a} 1}+\sum_{j=1}^{m}\left(M_{\mathrm{alaj}} \frac{\mathrm{d} i_{\mathrm{aj}}}{\mathrm{d} t}\right)+\sum_{i=1}^{n}\left(\frac{\mathrm{d}\left(M_{\mathrm{cial}} i_{\mathrm{c} i}\right)}{\mathrm{d} t}\right) \\
& 0=R_{\text {am }} i_{a m}+\sum_{j=1}^{m}\left(M_{\text {amaj }} \frac{\mathrm{d} i_{\text {aj }}}{\mathrm{d} t}\right)+\sum_{i=1}^{n}\left(\frac{\mathrm{d}\left(M_{\text {ciam }} i_{c i}\right)}{\mathrm{d} t}\right)
\end{aligned}
$$

$U_{\mathrm{c} i}$ in (1) becomes zero in (3) and $R_{\mathrm{c} i}$ in (1) need to be replaced by $R_{\mathrm{c} i}+R_{\mathrm{b} i}$ in (3) when the crowbar branch works. So (1) and (3) can be unified as (2). The determinations of the voltage matrix $U$ and the resistance matrix $R$ need to be according to the driving circuit state in the solved process of (2). The driving circuit state's judgment should be according to the changes of the capacitor's voltage or the driving current.

The axial electromagnetic force can be expressed as

$$
F_{\mathrm{z}}=\frac{1}{2} I_{1}^{\mathrm{T}} \hat{M} I_{2} \text { or } F_{\mathrm{z}}=\sum_{j=1}^{m} \sum_{i=1}^{n}\left(i_{\mathrm{a} j \mathrm{i}} i_{\mathrm{ci}} \frac{\mathrm{d} M_{\mathrm{ajci}}}{\mathrm{d} z}\right)
$$

The acceleration of the armature can be expressed as $a=F_{\mathrm{z}} / m$. Where, $m$ denotes the total mass of the armature and the load. 
When the initial speed is zero, the speed $v$ and the displacement $s$ can be respectively expressed as

$$
\begin{gathered}
v=\int_{0}^{t} a \mathrm{~d} t=\frac{1}{m} \int_{0}^{t} F_{\mathrm{z}} \mathrm{d} t, \\
s=\int_{0}^{t} v \mathrm{~d} t=\frac{1}{m} \int_{0}^{t}\left(\int_{0}^{t} F_{z} \mathrm{~d} t\right) \mathrm{d} t .
\end{gathered}
$$

Equation (2) and (4) to (6) form the DSM of MSSICL. It is necessary to be discretely processed in the solution course. The initial speed and the initial displacement of the next time step are calculated based on the initial speed, the initial displacement and the acceleration of the former time step. If the initial armature speed at $t$ moment is $v_{t}$, the armature speed $v_{t+\Delta t}$ at $t+\Delta t$ moment can be expressed as

$$
v_{t+\Delta t}=v_{t}+\int_{0}^{\Delta t} a_{t} \mathrm{~d} t=v_{t}+\frac{1}{m} \int_{0}^{\Delta t} F_{z-t} \mathrm{~d} t .
$$

Similarly, the armature displacement $s_{t+\Delta t}$ at $t+\Delta t$ moment can be expressed as

$$
s_{t+\Delta t}=s_{t}+\int_{0}^{\Delta t} v_{t} \mathrm{~d} t
$$

\subsection{Firing control criterion of MSSICL}

FCC is the determination criterion of the energized times or the energized locations. Therefore FCM of MSSICL includes FCC. The energized condition of MSSICL can be expressed as

$$
Z_{\mathrm{cm}}-Z_{\mathrm{ae}} \leq Z_{\text {slip }}+l_{\text {rise }},
$$

where, $Z_{\mathrm{ae}}$ is the armature tail's location, $Z_{\mathrm{cm}}$ is the center location of the driving coil, $Z_{\text {slip }}$ is the slip distance, $l_{\text {rise }}$ is the current rise length.

The energized location of the first stage need to be determined according to the critical FCC $Z_{\mathrm{cml}}-Z_{\text {ael }}=l_{\text {rise } 1}$ because slip distance of the first stage $Z_{\text {slip } 1}=0$. The energized locations of the other stages need to be determined according to (9). As far as MSSICL with given structure is concerned, the center location of the driving coil is fixed, and the armature location continuously changes in launch process. Let the location of the first stage driving coil's tail $Z_{\mathrm{cel}}=0$, the relationship between the armature tail's location and the armature displacement can be expressed as

$$
Z_{\text {ae }}=s+Z_{\text {ael }},
$$

where, Zae1 is the energized location of the first stage, $s$ is the armature displacement. $s$ can be obtained through (8). $Z_{\mathrm{ae}}$ can be obtained through (10). Whether the armature moves the proper energized location of each stage or not can be judged by (9).

\subsection{Solved method of FCM}

The initial state parameters including $U, R, I_{1}, I_{2}, \dot{I}_{1}, \dot{I}_{2}$, $M_{1}, M_{2}, \hat{M}, F_{\mathrm{z}}, v, a, Z_{\mathrm{ae} 1}, s, Z_{\mathrm{cm}}, Z_{\mathrm{slip}}, l_{\text {rise }}, m . U, m, Z_{\mathrm{ae}}$ can be given directly. $R, M_{1}, M_{2}, \hat{M}$ corresponding with the armature initial location need to be calculated. The initial values of $F_{\mathrm{z}}, v, a, s$ are zero. $Z_{\mathrm{cm}}$ can be determined according to the structure parameters of MSSICL. $Z_{\text {slip }}, l_{\text {rise }}$ for each stage are determined according to the experience. $I_{1}, I_{2}$ are both zero vectors. Considering only the first stage discharging and the other stage not discharging, the initial value of $\dot{I}_{1}$ and $\dot{I}_{2}$ can be set by $\dot{I}_{1}=\left[\begin{array}{llllllll}U_{\mathrm{cl}}(0) / L_{\text {ceq } 1} & 0 & \cdots & 0 & 0 & \cdots & 0\end{array}\right]^{\mathrm{T}}$, $\dot{I}_{2}=\left[\begin{array}{llllllll}0 & 0 & \cdots & 0 & U_{\mathrm{cl}}(0) / L_{\text {cal } 1} & \cdots & 0\end{array}\right]^{\mathrm{T}}$. Where, $L_{\mathrm{ceq} 1}$ is the equivalent inductance of the first stage or the selfinductance of the first stage with the armature. The current change ratios of the other stages at energized moment are also determined through the ratio of initial voltage to equivalent inductance.

Firstly, whether the energized condition is satisfied or not need to be judged by means of (9) in solving process of (2). If (9) is not satisfied, the corresponding component of current matrix keep zero. As far as some stage satisfying with the energized condition is concerned, the judgment of circuit state of the driving circuit is needed. If some stage driving circuit is in the state of before exchanged, (1) need to be solved. If some stage driving circuit is in the stage of after exchanged, the corresponding voltage component and resistance component need to be modified. If all stage driving circuits are in the stage of after exchanged, (3) need to be solved.

\section{ANALYSIS OF INFLUENCING FACTORS FOR FIRING CONTROL MODEL SOLVED PRECISION}

From the solved process of DSM, the correlative factors include the following point: (1)The calculative methods and the solved precision of $R, M$ and $\mathrm{d} M / \mathrm{d} z$ are the main influence factors; (2)The solved time step of the DSM has effect on the solved precision of FCM; (3)The judgment method of the driving circuit exchanged maybe also influence the solved precision of the DSM. From the FCC $Z_{\mathrm{cm}}-Z_{\mathrm{ae}} \leq Z_{\text {slip }}+l_{\text {rise }}$, the mainly influencing factors are the rationality of $Z_{\text {slip }}$ and $l_{\text {rise. }}$ As far as MSSICL with given structure is concerned, the values of $Z_{\text {slip }}$ and $l_{\text {rise }}$ are obtained by means of many modification combined with test validation.

\section{CONCLUSION}

The FCM is the key part of solving the MSSICL with super high velocity. The FCM is composed of the DSM and the FCC. The solved precision of the FCM is affected by many factors. The determination of the proper slip distance and current rise length need to be modified under the condition of considering many test results. 


\section{ACKNOWLEDGMENT}

This work was supported in part by the National Natural Science Foundation of China 51477181.

\section{REFERENCES}

1. R. J. Kaye, "Operational requirements and issues for coilgun electromagnetic launchers," IEEE Trans. Magn., vol.41, no.1, pp. 194-199, Jan. 2005.

2. I. R. Shokair, M. Cowan, R. J. Kaye, et al, "Performance of an induction coil launcher," IEEE Trans Magn., vol.31, no.1, pp. 510-515, Jan. 1995.

3. I. R. Shokair, M. Cowan, R. J. Kaye, et al, "Performance of an induction coil gun," Albuquerque, New Mexico, US: Sandia National Laboratories Report, SAND93-1358, 1993.

4. R. J. Kaye, B. Turman, M. Aubuchon, et al, "Induction coilgun for EM Mortar," in Proc. 16th IEEE Int. Pulsed Pulsed Power Conf., June. 2007, pp. 1810-1813.

5. Tao Zhang, wei Guo, Honghai Zhang, et al, "esign and testing of a 15-stage synchronous induction coilgu", 978-1-4673-0305-7/12/\$31.0062012.IEEE. 